2 Czuber-Dochan $W$, et al. The experience of fatigue in people with inflammatory bowel disease: an exploratory study. JAN 2013;69(9):1987-99

3 Colazzi P, Psychological research as a phenomenology views it. In Valle R, King M Eds. Existential Phenomenological Alternatives for Psychology. 1978;New York: New York University Press

Disclosure of Interest W. Czuber-Dochan Grant/research support from: Big Lottery Fund managed by Crohn's and Colitis UK, C. Norton Grant/research support from: Big Lottery Fund managed by Crohn's and Colitis UK, S. Berliner Conflict with: Trustee Crohn's and Colitis UK, F. Bredin Grant/research support from: Big Lottery Fund managed by Crohn's and Colitis UK, M. Darvell Employee of: Crohn's and Colitis UK, A. Forbes Grant/ research support from: Big Lottery Fund managed by Crohn's and Colitis UK, M. Gay Conflict with: Vice-Chair and Trustee Crohn's and Colitis UK, I. Nathan Grant/research support from: Big Lottery Fund managed by Crohn's and Colitis UK, E. Ream: None Declared, H. Terry Employee of: Crohn's and Colitis UK.

\section{PWE-121 DEVELOPMENT AND PSYCHOMETRIC TESTING OF AN INFLAMMATORY BOWEL DISEASE FATIGUE (IBD-F) PATIENT SELF-ASSESSMENT SCALE}

${ }^{1}$ WJ Czuber-Dochan* ${ }^{*}{ }^{1} \mathrm{C}$ Norton, ${ }^{2} \mathrm{~S}$ Berliner, ${ }^{3} \mathrm{~F}$ Bredin, ${ }^{2} \mathrm{M}$ Darvell, ${ }^{4} \mathrm{~A}$ Forbes, ${ }^{2} \mathrm{M}$ Gay, ${ }^{4}$ I Nathan, ${ }^{1} \mathrm{E}$ Ream, ${ }^{2} \mathrm{H}$ Terry, ${ }^{5} \mathrm{P}$ Bassett. ${ }^{1}$ Florence Nightingale School of Nursing and Midwifery, King's College London, London, UK; ${ }^{2}$ Crohn's and Colitis UK, St Albans, UK; ${ }^{3}$ Gastroenterology, The Queen Elizabeth Hospital NHS Trust, King's Lynn, UK; ${ }^{4}$ Gastroenterology and Clinical Nutrition, University College London, London, UK; ${ }^{5}$ Statsconsultancy, Amersham, UK

\subsection{6/gutjnl-2014-307263.381}

Introduction Fatigue is one of the main symptoms of inflammatory bowel disease (IBD) and is frequently reported by people in both active and quiescent disease. Different fatigue assessment scales have been used to measure fatigue, but none has been developed or tested in IBD. ${ }^{1}$ This study aimed to develop a new fatigue scale specific to the needs and experiences of people with IBD.

Methods A sequential mixed methods design was used: a qualitative phase (Phase 1) to assess patients' experience of fatigue and four mixed qualitative-quantitative phases (Phase 2-5) to refine the scale and to assess its psychometric properties. Phase 1-4 participants were purposively selected from a group of volunteers who self reported their fatigue, and participants for Phase 5 were randomly selected from the Crohn's and Colitis UK members database.

Results 567 people participated in the 5 phases. The resulting IBD-F questionnaire has 3 sections: Section 1 Fatigue Assessment; Section 2 Fatigue Impact on Daily Activities; Section 3 Additional Comments about Fatigue. Initial validation suggests that the questionnaire has good face and content validity and acceptable to excellent test-retest stability (ICC 0.74 for section 1 and 0.83 for section 2) and a high degree of internal consistency with Cronbach's alpha value of over 0.9 .

Conclusion The participants in the study confirmed that fatigue in IBD is burdensome. Items generated and refined by people with IBD-fatigue reflect their experience and form the basis of this new IBD-fatigue scale, which is psychometrically robust and its reliability falls within statistically acceptable ranges. The fatigue scores obtained by the newly developed, disease specific IBD-F self-assessment scale strongly correlated with the existing fatigue scales (MFI and MAF) developed with other diseases., ${ }^{2,3}$ The scale can be used by patients and practitioners to assess severity and impact of fatigue in people with IBD. An electronic copy of IBD-F scale can be accessed through website link http:// www.fatigueinibd.co.uk from July 2014.

\section{REFERENCES}

1 Whitehead L, The measurement of fatigue in chronic illness: a systematic review of unidimensional and multidimensional fatigue measures. J of Pain and Symptom Manag. 2009;37(1):107-28

2 Smets $\mathrm{EM}$, et al. The Multidimensional Fatigue Inventory (MFI) psychometric qualities of an instrument to assess fatigue. J of Psychosom Research. 1995;39:315-25

3 Belza B, et al. Correlates of fatigue in older adults with rheumatoid arthritis. Nur Research. 1993;42(2):93-9

Disclosure of Interest W. Czuber-Dochan Grant/research support from: Big Lottery Fund managed by Crohn's and Colitis UK, C. Norton Grant/research support from: Big Lottery Fund managed by Crohn's and Colitis UK, S. Berliner Conflict with: Trustee Crohn's and Colitis UK, F. Bredin Grant/research support from: Big Lottery Fund managed by Crohn's and Colitis UK, M. Darvell Employee of: Crohn's and Colitis UK, A. Forbes Grant/ research support from: Big Lottery Fund managed by Crohn's and Colitis UK, M. Gay Conflict with: Vice-Chair and Trustee Crohn's and Colitis UK, I. Nathan Grant/research support from: Big Lottery Fund managed by Crohn's and Colitis UK, E. Ream: None Declared, H. Terry Employee of: Crohn's and Colitis UK, P. Bassett: None Declared.

\section{PWE-122 DETERMINANTS OF WEIGHT LOSS PRIOR TO DIAGNOSIS IN INFLAMMATORY BOWEL DISEASE: A RETROSPECTIVE OBSERVATIONAL STUDY}

C Alexakis, Y Elsherrif*, M Mendall. Croydon University Hospital, London, UK

\subsection{6/gutjnl-2014-307263.382}

Introduction Weight loss is a recognised presenting feature of Inflammatory Bowel Disease (IBD) and considered as marker of malnutrition and disease severity. This is well established in children with IBD, however in adults, evidence based data is lacking. There is little in the literature characterising weight loss prior to formal diagnosis, or defining the disease factors associated with it.

Methods Patients with IBD attending outpatient clinic were provided with a questionnaire enquiring into various aspects of their disease. Percentage Body Mass Index loss prior to diagnosis was calculated for each subject. Patients were sub-categorised into groups according to severity of $\%$ BMI loss (insignificant $<5 \%$, moderate $5-10 \%$, severe $10-20 \%$, extreme $>20 \%$ ), and disease and patient factors associated with weight loss were determined. Results 494 subjects were recruited. 237 had Crohn's Disease (CD); 257 had Ulcerative Colitis (UC). Mean\%BMI loss prior to diagnosis was greater in CD (CD 9.76\% vs UC 7.63, $\mathrm{p}=0.02$ ). Increasing age at diagnosis was inversely associated with weight loss prior to diagnosis in UC $(-0.1$ per year of age, $95 \%$ CI $0.17--0.03, p=0.004)$, and CD $(-0.15$ per year of age, $95 \%$ CI $-0.23--0.06, p=0.003)$. In CD, patients with prior appendectomy had reduced risk of weight loss (HR 0.38, $\mathrm{p}=0.014)$. Ileal disease was a risk factor in patients with extreme weight loss.

Conclusion Weight loss is a significant problem for many IBD patients at presentation, especially in younger age and Crohn's disease with ileal involvement. Improved awareness of the presenting features of IBD should encourage wider use of malnutrition screening tools and earlier investigative tests to uncover patients at risk. Appendectomy confers a protective effect in CD. Disclosure of Interest None Declared. 